\title{
Magnetic Instability in HTSC films
}

\author{
P. Brïll, V. Bujok, J. Boneberg, S. Herminghaus and P. Leiderer
}

Fakultăt für Physik, Universität Konstanz, 7750 Konstanz, Germany

We have investigated the short time dynamics in the magnetic flux distribution of thin $\mathrm{YBa}_{2} \mathrm{Cu}_{3} \mathrm{O}_{7-\mathrm{x}}$ films by means of time resolved magnetooptics. Local heating of the superconductor with a focussed laser pulse in a region with high shielding currents leads to an instability in the magnetic flux distribution. A pump probe technique allowed to visualize changes in the flux distribution on a nanosecond time scale. The instability develops in two steps giving rise to additional flux penetration and to a branching of the flux front into the superconductor.

\section{Introduction}

Thin superconducting films in transverse magnetic fields are close to being unstable. In the case of high critical current densities (equivalent to high pinning forces) large gradients can be established in the field distribution. This gives rise to a strong non-equilibrium, and small thermal perturbations can lead to an instability which results in drastical changes in the magnetic field distribution. In contrast to earlier studies of flux jumps, we have artificially triggered the instability by a local perturbation of the superconducting state, using a focussed laser pulse. The measurements yield propagation speeds of the flux fronts 5 orders of magnitude larger than for massive samples.

\section{Experiment}

The $\mathrm{YBa}_{2} \mathrm{Cu}_{3} \mathrm{O}_{7-x}$ sample was an epitaxial coriented film with a thickness of $300 \mathrm{~nm}$, deposited on $1.1 \mathrm{~cm}^{2} \mathrm{LaAlO}_{3}$ substrates by laser ablation [1]. The measurements were carried out in an optical cryostat equipped with a superconducting magnet. The direction of the magnetic field was perpendicular to the sample plane. In order to detect the flux distribution in the sample we used a reflection magnetooptic technique, with a EuS indicator film as described earlier [2]. The sample was zero-field cooled and immersed in superfluid ${ }^{4} \mathrm{He}$ at $1.8 \mathrm{~K}$. As a magnetic field was applied, the evolution of the flux distribution in the sample was recorded with a video camera using a standard magnetooptic set-up with a spacial resolution of $10 \mu \mathrm{m}$. The field was typically increased up to a few $10^{-2} \mathrm{~T}$, where flux entering from the sample edges roughly filled several ten percent of the film area, leaving a large portion of the sample in the Meissner state [3]. In order to perturb this state and to initiate the magnetic instability, a pulse of a frequency doubled $\mathrm{Nd}$ :YAG laser $(\lambda=532 \mathrm{~nm}$, FWHM 7 ns, energy up to $30 \mathrm{~mJ}$ ) was focussed onto the superconducting film from the back side through the polished substrate. The laser focus with a diameter of $50 \mu \mathrm{m}$ could be positioned arbitrarily on the sample surface. At the laser energies used here the sample temperature in the focus, although not being measured directly, supposedly rose above the critical temperature of the superconductor $\left(T_{c}=92 \mathrm{~K}\right)$.

A pump probe technique was applied to achieve the high temporal resolution necessary to determine the spreading velocity of the flux fronts. Part of the Nd:YAG beam was separated by a beam splitter, passed through a variable delay line of several ten $\mathrm{ns}$, and frequency shifted to $\lambda=635 \mathrm{~nm}$ (details are described in Ref.[4]). This pulse was fed into the magnetooptic light path to illuminate the EuS film at a well defined time delay some ten nanoeconds after the instability had been nucleated. The comparison of the extension of the magnetic structure in this snapshot with the final flux distribution allowed to determine the spreading velocity.

\section{Results and Discussion}

If a magnetic field is applied perpendicular to a film with a thickness comparable to the London penetration depth $(\sim 150 \mathrm{~nm})$ large shielding currents flow even in the Meissner phase. Fig. 1 shows the result of a time resolved experiment. The frames correspond to a $4 \times 4 \mathrm{~mm}^{2}$ fraction of the superconducting sample. The figure displays a sequence of pictures taken before (1a), 56ns after (1b) and 5s after (1c) the laser pulse. A comparison of figs. $1 \mathrm{~b}$ and $1 \mathrm{c}$ shows that already after $t=56 \mathrm{~ns}$ the flux

Konstanzer Online-Publikations-System (KOPS)

URL: http://www.ub.uni-konstanz.de/kops/volltexte/2007/3071/

URN: http://nbn-resolving.de/urn:nbn:de:bsz:352-opus-30710 
a)

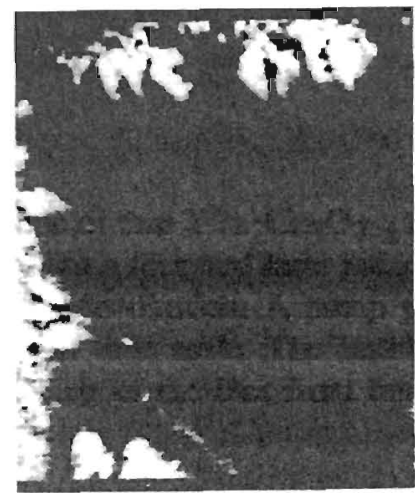

b)

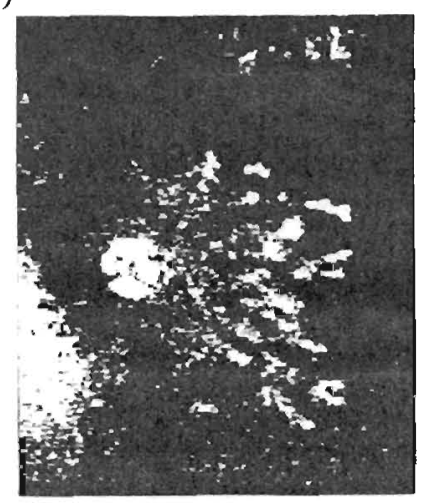

c)

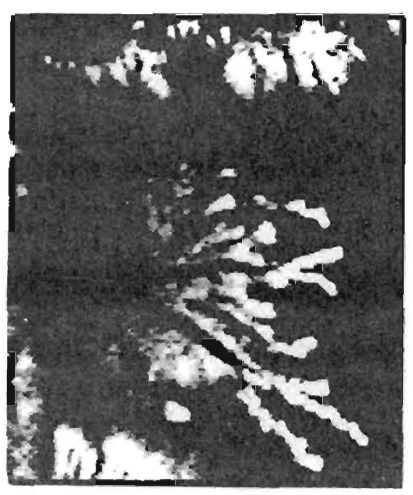

Fig. 1: Development of the magnetic instability in the magnetic field distribution. a) before nucleation, b) $56 \mathrm{~ns}$ after nucleation with a focussed $\mathrm{N} d$ : YAG laser pulse c) final field distribution.

structure has nearly reached its final size. Nevertheless, some of the branches are not yet fully developed and apparently still are to grow a few tenths of a mm (compare especially the bottom right part of the structure in fig. $1 \mathrm{~b}$ and $1 \mathrm{c}$ ).

The flux motion triggered by the laser pulse actually develops in two steps: i) First, a perturbation grows in the Meissner phase around the nucleation spot in a roughly isotropic way, forming a normal conducting region where flux, accumulated at some weak parts of the film before, is afterwards distributed homogeneously within the perturbed area. ii) As soon as this region makes contact with the surrounding extemally applied field at some point, massive penetration takes place. When this extremely fast penetrating flux front reaches the border to the still superconducting region in the Meissner phase, avalanche-like penetration of external flux sets in, and branches start to spread out from the rim of the homogeneous area.

A series of such recordings with variable delay times has been used to investigate the growth dynamics of the flux structures in more detail. These studies reveal that the spatially homogeneous field distribution in the transient normal conducting region develops extremely fast, on a time scale which is not even accessible with our temporal resolution of $10 \mathrm{~ns}$. We therefore can only estimate that the speed at which the front of this phase propagates is larger than $2 \cdot 10^{5} \mathrm{~m} / \mathrm{s}$. The propagation speed of the branches, on the other hand, was small enough that it could be determined with our technique. The data yield $\langle\mathrm{v}\rangle=(5 \pm 2) \cdot 10^{4} \mathrm{~m} / \mathrm{s}$, which, for comparison, is an order of magnitude higher than the velocity of sound in $\mathrm{YBa}_{2} \mathrm{Cu}_{3} \mathrm{O}_{7-x}$ [5].

In summary, we have studied the evolution of a novel instability nucleated by a laser pulse in thin superconducting films exposed to a static magnetic field. We find two regimes, first a process where the perturbation leads to a homogeneous redistribution of flux over a certain region of the sample from the outside. Then it further penetrates into the Meissner phase in the shape of branches which propagate at a speed of about $5 \times 10^{4} \mathrm{~m} / \mathrm{s}$. The experiments have been performed with a high- $\mathrm{T}_{c}$ superconductor, but we expect similar phenomena to occur also for conventional hard typeII superconductors.

\section{Acknowledgements}

We acknowledge helpful discussions with $\mathrm{A}$. L. Rakhmanov and E. H. Brandt. This work was supported by BMFT grant 13 N5705 and Schwerpunktprogramm Land Baden Württemberg.

\section{References}

[1] J. Fröhlingsdorf, W. Zander, B. Stritzker, Solid State Commun. 67 (1988) 965

[2] P. Brïll, D. Kirchgässner, P. Leiderer, Physica C 182 (1991) 339

[3] H. Theuss, A. Forkl, H. Kronmüller, Physica C 190 (1992) 345

[4] V. Bujok, P. Brüll, J. Boneberg, S. Herminghaus, P. Leiderer, submitted to Appl. Phys. Lett.

[5] T. J. Kim, J. Kowalewski, W. Assmus, W. Grill, Z. Phys. B - Condensed Matter 78 (1990) 207 